\title{
Prevalence of the Metabolic Syndrome and Its Components among Obese Men and Women in Italy
}

\author{
Claudio L. Lafortuna ${ }^{a}$ Fiorenza Agostic Alessandra De Col ${ }^{c}$ Franca Pera $^{d}$ \\ Fulvio Adorni $^{b} \quad$ Alessandro Sartorio $^{c, d}$ \\ astituto di Bioimmagini e Fisiologia Molecolare, ${ }^{b}$ Istituto di Tecnologie Biomediche, Consiglio

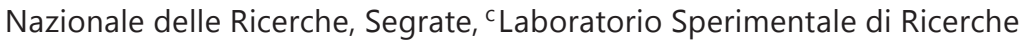 \\ Auxo-endocrinologiche, dDivisione Malattie Metaboliche, Istituto Auxologico Italiano, IRCCS, \\ Milano and Piancavallo, Italy
}

\author{
Key Words \\ Obesity $\cdot$ Blood pressure $\cdot \mathrm{HDL}$ cholesterol $\cdot$ Triglycerides $\cdot$ Fasting plasma glucose
}

\begin{abstract}
Objective: Assessment of the prevalence of the metabolic syndrome (MetS) and its components in Italian obese individuals. Methods: Cross-sectional estimate of MetS prevalence using the IDF definition in 635 obese (BMI $\geq 35 \mathrm{~kg} / \mathrm{m}^{2}$ ) individuals ( $286 \mathrm{men}, 349$ women) aged 19-78 years hospitalised for mass excess treatment. Results: MetS prevalence was significantly $(\mathrm{p}<0.001)$ higher in males $(75.9 \%)$ than in females $(61.9 \%)$, due to the increased prevalence of $4 / 5$ involved abnormalities. A multivariate binary logistic regression analysis disclosed a contributory independent effect of ageing ( $p<0.01-0.001)$, but not of BMI, whereas large waist circumference $(p<0.01)$ and high waist-to-hip ratio $(p<0.01)$ contributed to MetS prevalence only in women. Affected individuals were significantly older (males $p=0.022$; females $p<0.001)$, affected men being younger than affected women $(p<0.01)$. The prevailing clinical picture included all abnormalities ( $21.2 \%$ in men, $22.2 \%$ in women); in minimal trait MetS (3/5 abnormalities), the prevailing combination was, beside central obesity, hypertension-hyperglycaemia in men (12.4\%), and hypertension-dyslipidemia in women (19.4\%). Conclusions: MetS is a highly prevalent condition among Italian obese individuals. Prevalence and combinations of involved abnormalities differ considerably between genders and suggest a different pathophysiology of the MetS in men and women, with possible relevance for prevention and treatment of the condition.




\section{Introduction}

The metabolic syndrome (MetS) is a multifactorial clinical entity with a very high (and still rising) prevalence in the general population of all countries investigated, involving an increased risk for mortality from cardiovascular disease and other causes [1-4]. The abnormalities that tend to cluster include raised arterial blood pressure (BP), reduced plasma levels of high-density lipoprotein cholesterol (HDL-CH), raised triglycerides (TG) plasma levels, raised fasting plasma glucose (FPG) concentration and obesity. In particular, central obesity has been recognised as a key element sustaining this syndrome [5], to the point that the International Diabetes Federation (IDF) recently included waist circumference (WC) above a critical cut-off value as a mandatory criterion for the diagnosis of the MetS [6].

In fact, adipose tissue excess is considered the driving factor in the development of the MetS, the prevalence increasing exponentially from the normal weight range to overweight and obesity [7]. A dysregulated production of multiple bioactive molecules from adipose tissue adversely affects different metabolic processes and ultimately participates in the pathogenesis of obesity-associated conditions such as insulin resistance, dyslipidaemia and hypertension $[8,9]$, which are all key components of the MetS.

Nonetheless, in spite of the abundance of studies evaluating the prevalence of the MetS among specific cohorts with different characteristics and habits [1], the investigations restricted to samples of only obese individuals of both genders are surprisingly scanty, although most large epidemiological surveys included also individuals with BMI in the range of obesity [2-4, 7]. We recently evaluated the prevalence and the clustering of the components of the MetS in homogeneous cohorts of obese women and reported in this gender a definite association of the pattern of lipid accumulation, a sexually regulated feature of body fat distribution, with disturbed glucose homeostasis and dyslipidaemia [10, 11]. Thus, due also to gender-related differences in the pathological mechanisms underlying MetS and its related cardiovascular risks [12], obese males may possibly display a different characterisation of the MetS profile.

Therefore the objectives of the present study in obese European men and women were to estimate in the two genders: i) the prevalence of the MetS using the diagnostic criteria defined by the IDF [6], ii) the effect of age, BMI, WC and relative distribution of adiposity on the prevalence of the MetS and iii) the prevalence of each MetS component as well as the predominant combinations.

\section{Patients and Methods}

\section{Subjects}

The cohort of the study consisted in 635 consecutive obese individuals ( 286 males, 349 females) aged 19-78 years. All subjects were in-patients with obesity class II and III (BMI $\geq 35 \mathrm{~kg} / \mathrm{m}^{2}$ ) admitted for treatment of adiposity excess. The Ethical Committee of Istituto Auxologico Italiano approved the study, and the patients gave their written informed consent.

Evaluation of Subjects

All measurements were performed before the beginning of the protocol aimed at body mass reduction. At hospital admission, height and weight were measured, and BMI was calculated. WC was measured at the narrowest place (or, if indeterminable, at mid-distance) between iliac crest and rib cage, and hip circumference at the at the widest point over the greater trochanter [13]. From these measurements, waist-to-hip ratio (WHR) was calculated. Three BP determinations were performed on different days, and their value was averaged. Blood samples were collected after an overnight fast in standard tubes; TG and 
DOI: $10.1159 / 000336700$

Published online: March 2, 2012

(C) 2012 S. Karger GmbH, Freiburg

Lafortuna et al.: Prevalence of the Metabolic Syndrome and Its Components among

Obese Men and Women in Italy

Table 1. The average characteristics of 635 obese men and women analysed in the study

\begin{tabular}{|c|c|c|c|c|c|c|c|}
\hline & \multicolumn{3}{|c|}{$\operatorname{Men}(\mathrm{N}=286)$} & \multicolumn{3}{|c|}{ Women $(\mathrm{N}=349)$} & \multirow[t]{2}{*}{$\mathrm{P}$} \\
\hline & mean (SD) & median & range & mean (SD) & median & range & \\
\hline Age, years & $46.7(14.3)$ & 47.0 & $19-78$ & $48.5(13.3)$ & 49.0 & $19-78$ & 0.147 \\
\hline Height, m & $1.73(0.08)$ & 1.73 & $1.45-1.93$ & $1.58(0.07)$ & 1.59 & $1.41-1.80$ & $<0.001$ \\
\hline Body weight, kg & $131.4(22.0)$ & 129.1 & $81.4-210.0$ & $109.9(18.2)$ & 106.5 & 78.7-201.6 & $<0.001$ \\
\hline BMI, kg/m² & $44.1(6.1)$ & 42.8 & $35.2-69.0$ & $44.0(6.5)$ & 42.8 & $35.1-83.1$ & 0.661 \\
\hline Waist circumference, $\mathrm{cm}$ & $134.6(13.0)$ & 133.0 & $95.0-180.0$ & $121.0(13.0)$ & 120.0 & $88.0-179.0$ & $<0.001$ \\
\hline WHR & $1.03(0.08)$ & 1.03 & $0.64-1.60$ & $0.91(0.10)$ & 0.91 & $0.66-1.40$ & $<0.001$ \\
\hline Triglycerides, mg/dl & $160.2(82.8)$ & 146.0 & $56-674$ & $136.0(65.3)$ & 122.0 & $37-535$ & $<0.001$ \\
\hline HDL-cholesterol, mg/dl & $40.3(9.9)$ & 39.0 & $12-75$ & $50.5(12.2)$ & 48.0 & $24-91$ & $<0.001$ \\
\hline Fasting plasma glucose, mg/dl & $102.4(31.1)$ & 93.0 & $59-261$ & $98.0(31.5)$ & 89.0 & $56-316$ & 0.080 \\
\hline Fasting serum insulin, $\mu \mathrm{U} / \mathrm{ml}$ & $15.9(8.2)$ & 14.0 & $2-61$ & $11.9(8.0)$ & 10.5 & $0-78$ & $<0.001$ \\
\hline HOMA-IR & 4.15 (2.89) & 3.47 & $0.41-24.60$ & $2.97(2.45)$ & 2.36 & $0.38-28.01$ & $<0.001$ \\
\hline Systolic blood pressure, $\mathrm{mm} \mathrm{Hg}$ & $131.2(15.8)$ & 130.0 & $100-220$ & $127.7(15.0)$ & 125.0 & $100-215$ & 0.001 \\
\hline Diastolic blood pressure, $\mathrm{mm} \mathrm{Hg}$ & $79.2(9.7)$ & 80.0 & $60-140$ & $76.7(8.0)$ & 80.0 & $50-100$ & $<0.001$ \\
\hline
\end{tabular}

Statistical significance of the difference between genders is assessed by means of Mann-Whitney U-test for independent samples.

HDL-CH as well as FPG were immediately measured with enzymatic colorimetric methods (Hitachi Instruments, Tokyo, Japan) after appropriate processing. Fasting serum insulin (FSI) was determined by a two-site chemiluminescent immunometric assay (DPC Immulite 2000, Siemens, Munich). Insulin resistance was estimated using the homeostatic model assessment of insulin resistance (HOMA-IR) as HOMA-IR $=($ FSI $\times$ FPG $) / 22.5$, where FSI is expressed in $\mu \mathrm{U} / \mathrm{ml}$ and FPG in mmol/l [14]. Table 1 shows the main characteristics of the participating subjects.

At their enrolment, $56.1 \%$ and $54.2 \%$ of men and women were receiving treatment for hypertension, $27.0 \%$ and $20,7 \%$ for hyperglycaemia, or $13.4 \%$ and $12.6 \%$ for dyslipidaemia, respectively. Considering a HOMA-IR cut-off value of 2.6 [14], 70\% of males and $44 \%$ of females had insulin resistance.

According to the IDF definition [8], the patients were considered to have MetS if they had central obesity (WC $\geq 94 \mathrm{~cm}$ in males and $\geq 80 \mathrm{~cm}$ in females) plus two or more of the following factors: i) raised TG level ( $\geq 150 \mathrm{mg} / \mathrm{dl}$ (1.7 mmol/l) or specific treatment for this lipid abnormality); ii) reduced HDL-CH $(<40 \mathrm{mg} / \mathrm{dl}(1.03 \mathrm{mmol} / \mathrm{l})$ in males and $<50 \mathrm{mg} / \mathrm{dl}(1.29 \mathrm{mmol} / \mathrm{l})$ in females or specific treatment for this lipid abnormality); iii) raised BP (systolic BP $\geq 130 \mathrm{~mm} \mathrm{Hg}$ or diastolic BP $\geq 85 \mathrm{~mm} \mathrm{Hg}$ or treatment of previously diagnosed hypertension); iv) raised FPG concentration ( $\geq 100 \mathrm{mg} / \mathrm{dl}$ (5.6 mmol/l) or previously diagnosed type 2 diabetes).

\section{Statistical Methods}

All values are given as means \pm SD. Comparison of the MetS prevalence and its single components between genders was performed by means of Pearson's chi square test. Mean values were compared using Student's t-test for unpaired data. The effect of age, BMI, WC and WHR on the prevalence of the MetS was evaluated with a multivariate binary logistic regression analysis. To this aim, the subjects were stratified into three groups of age $(<40,40-54, \geq 55$ years), and two groups with median value as a cut-off for BMI $\left(43 \mathrm{~kg} / \mathrm{m}^{2}\right.$ in both genders), for WC (133 cm in males and 120 in females) and for WHR (1.03 in males and 0.91 in females).

P values less than 0.05 were considered statistically significant. Statistical software package SPSS 14 (SPSS Inc., Chicago, IL, USA) for Windows was used for all analyses. 


\section{Results}

Using the new IDF criteria for adults [8], the overall prevalence of the MetS in the whole cohort of these obese individuals was $68.2 \%$ and was significantly $(p<0.001)$ higher in men $(75.9 \%)$ than in women (61.9\%). While central obesity affected all men and women within the cohort, the other single components were present to a different degree in the two genders. Raised BP (males 80.4\% vs. females 69.3\%; p <0.001), as well as raised TG level (51.8\% vs. $39.3 \%$; $\mathrm{p}<0.01)$ and raised FPG $(44.1 \%$ vs. $32.4 \%$; $\mathrm{p}<0.01)$ were all significantly more prevalent in men while no significant difference between genders was detected in prevalence of reduced HDL-CH (56.6\% vs. 61.9\%; $\mathrm{p}=0.180$ ).

The distribution of the number of concomitant abnormalities was significantly different between men and women $(\mathrm{p}<0.01)$. While women had a higher prevalence of a single or two concomitant components, men had a higher prevalence of clinical pictures entailing three or more abnormalities. Individuals with MetS were significantly older than those without MetS within both genders (males $47.8 \pm 14.1$ vs. $43.3 \pm 14.7$ years, $\mathrm{p}=0.022$; females $51.7 \pm 12.5$ vs. $43.2 \pm 13.0$ years, $\mathrm{p}<0.001$ ), but men with MetS were also significantly younger than women with MetS $(\mathrm{p}<0.01)$, suggesting that women achieve the same metabolic derangement of men at an older age. Men and women without MetS have similar age.

The prevalence of the MetS estimated within the different strata of age, BMI, WC and WHR is presented in table 2 for men and women, along with the independent effect of each factor.

The prevalence of the single abnormalities of the MetS in the different strata of age, BMI and WHR is presented in fig. 1 for males and females. While the prevalence of raised BP, TG and FPG has a defined tendency to increase with age, reduced HDL-CH prevalence appears levelled in both genders. On the contrary, a higher WHR exerts a positive effect on the prevalence of all abnormalities only in women; in men this effect exerted by fat distribution is less evident. No systematic trend in the prevalence of the single abnormalities can be detected for effect of BMI in both genders.

In fig. 2 the prevalence of the different combinations of abnormalities in males and females without (panel A) and with (panel B) MetS are summarised. In both men and women without MetS the leading combination entailed central obesity and raised BP (males 56.5\%, females 39.8\%). Among individuals with MetS, males with three abnormalities principally presented (beside central obesity) high BP and raised FPG (31.4\% of males with three abnormalities and $12.4 \%$ of all males with MetS); in females the leading combination entailed high BP and low HDL-CH (47.2\% of females with three abnormalities and 19.4 of all females with MetS). Among those with four metabolic abnormalities, the leading combination for both genders was high BP, low HDL-CH and raised TG (43.5\% of males with four abnormalities and $17.1 \%$ of all males with MetS; $50.6 \%$ of females with four abnormalities and $18.5 \%$ of all females with MetS). All five abnormalities were present in $21.2 \%$ and $22.2 \%$ of men and women with MetS, respectively.

The effect of age on the prevalence of the principal combinations of abnormalities is presented in fig. 3. In spite of a certain variability among genders in absolute prevalence of combinations, the trend of prevalence for effect of age is similar in men and women. Combinations including raised FPG appeared to increase with age while combinations with dyslipidaemic abnormalities appeared to decrease with age. In fact, both men and women with raised FPG were significantly older than those without raised FPG (males $52.3 \pm 11.8$ vs. $41.9 \pm 14.7$ years, $\mathrm{p}<0.001$; females $56.1 \pm 11.2$ vs. $47.5 \pm 12.3$ years, $\mathrm{p}<0.001$ ). Males were significantly younger than females of the corresponding group (with raised FPG $\mathrm{p}<$ 0.001; without raised FPG p < 0.01). 
Table 2. The prevalence of the MetS within the different strata of age, BMI, waist circumference (WC), and waist-to-hip ratio (WHR) in obese men and women from the investigated cohort and the results of a multivariate binary logistic regression

\begin{tabular}{|c|c|c|c|c|c|c|}
\hline & $\mathrm{N}$ & Prevalence, \% & AOR & CI (95\%low) & CI (95\%high) & $\mathrm{p}$ \\
\hline \multicolumn{7}{|l|}{ Men } \\
\hline Total & 286 & 75.9 & & & & \\
\hline \multicolumn{7}{|c|}{ Age, years } \\
\hline$<40$ & 91 & 63.7 & 1 & & & \\
\hline $40-54$ & 103 & 79.6 & 2.278 & 1.187 & 4.370 & $<0.05$ \\
\hline$\geq 55$ & 92 & 83.7 & 3.478 & 2.224 & 6.405 & $<0.01$ \\
\hline \multicolumn{7}{|c|}{ BMI, $\mathrm{kg} / \mathrm{m}^{2}$} \\
\hline$<43$ & 147 & 72.1 & 1 & & & \\
\hline$\geq 43$ & 139 & 79.9 & 1.544 & 0.747 & 3.193 & 0.241 \\
\hline \multicolumn{7}{|c|}{ WC, class* } \\
\hline Low & 142 & 71.8 & 1 & & & \\
\hline High & 144 & 79.9 & 1.352 & 0.652 & 2.803 & 0.417 \\
\hline \multicolumn{7}{|c|}{ WHR, class** } \\
\hline Low & 145 & 74.4 & 1 & & & \\
\hline High & 141 & 77.3 & 1.067 & 0.600 & 1.898 & 0.824 \\
\hline \multicolumn{7}{|l|}{ Women } \\
\hline Total & 349 & 61.9 & & & & \\
\hline \multicolumn{7}{|l|}{ Age, years } \\
\hline$<40$ & 93 & 39.8 & 1 & & & \\
\hline $40-54$ & 136 & 61.8 & 2.149 & 1.208 & 3.823 & $<0.01$ \\
\hline$\geq 55$ & 120 & 79.2 & 4.416 & 2.331 & 8.367 & $<0.001$ \\
\hline \multicolumn{7}{|c|}{ BMI, $\mathrm{kg} / \mathrm{m}^{2}$} \\
\hline$<43$ & 177 & 57.6 & 1 & & & \\
\hline$\geq 43$ & 172 & 66.3 & 1.068 & 0.615 & 1.856 & 0.815 \\
\hline \multicolumn{7}{|c|}{ WC, class* } \\
\hline Low & 174 & 47.7 & 1 & & & \\
\hline High & 175 & 76.0 & 2.261 & 1.258 & 4.063 & $<0.01$ \\
\hline \multicolumn{7}{|c|}{ WHR, class** } \\
\hline Low & 174 & 47.7 & 1 & & & \\
\hline High & 175 & 76.0 & 2.206 & 1.313 & 3.706 & $<0.01$ \\
\hline
\end{tabular}

\section{Discussion}

Using the new diagnostic criteria deriving from the recent IDF consensus statement [6], in this cohort of Italian obese individuals seeking medical help for treatment of their mass excess, $62 \%$ of women and $76 \%$ of men aged $19-78$ years met the criteria for MetS, with an overall prevalence of $68 \%$. In both genders a contributory independent effect was exerted by advancing age, but not by the degree of obesity, as represented by BMI, whereas large abdominal circumference and high WHR had a positive effect on MetS prevalence only among women. 

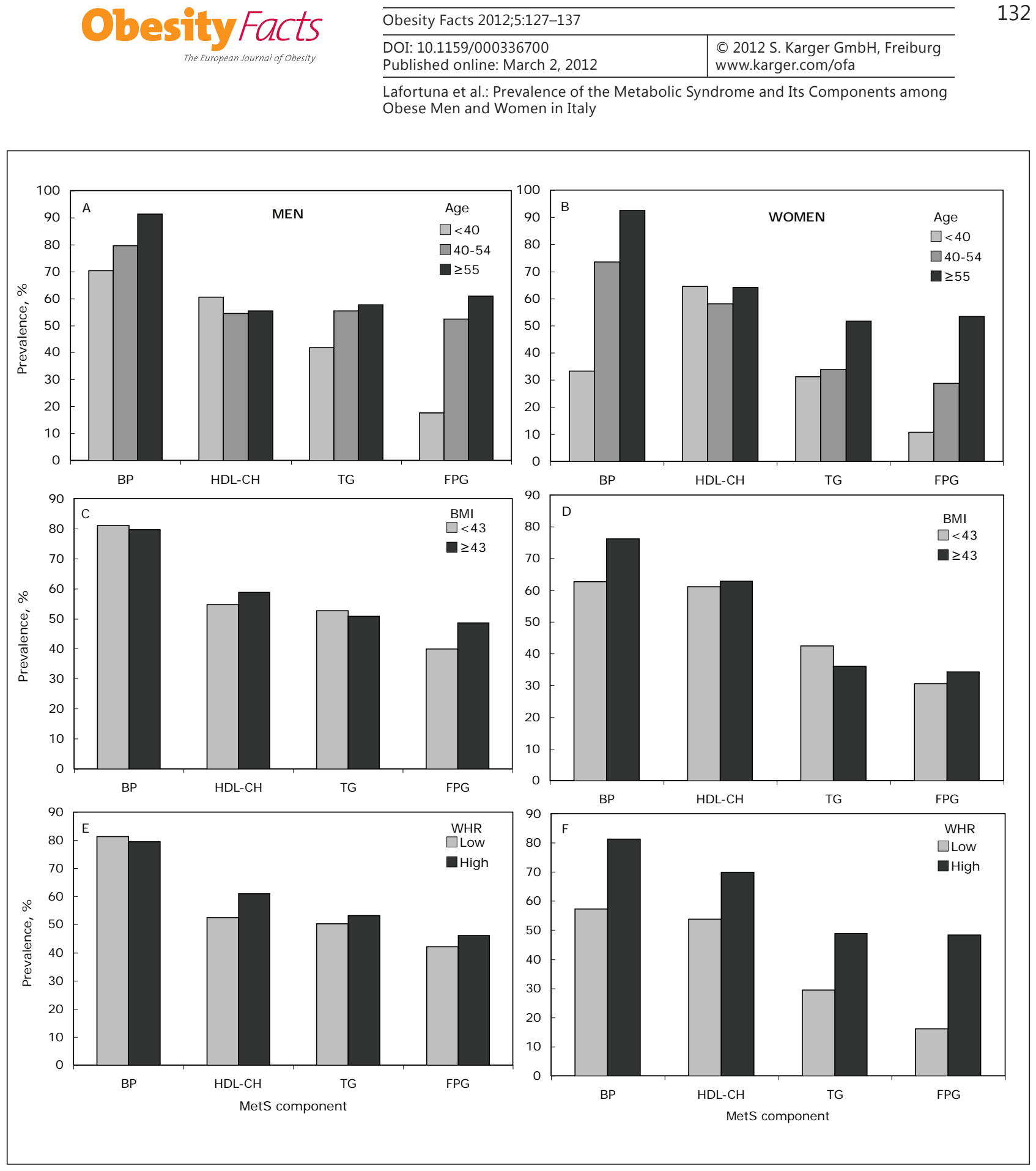

Fig. 1. The prevalence of the five abnormalities of the metabolic syndrome for men and women within the different strata of age (panels $\mathbf{A}$ and $\mathbf{B}$ ), BMI (panels $\mathbf{C}$ and $\mathbf{D}$ ) and WHR (panels E and $\mathbf{F}$ ). BP = raised blood pressure or treatment of previously diagnosed hypertension; HDL-CH = reduced HDL cholesterol or specific treatment for this lipid abnormality; TG = raised triglycerides level or specific treatment for this lipid abnormality; FPG = raised fasting plasma glucose concentration or previously diagnosed type 2 diabetes.

A very consistent finding in the literature is the highly age-dependent prevalence of the MetS worldwide. In agreement with previous findings of several studies on national populations from different countries [2-4, 7], advancing age is an important determinant of the syndrome also in the obese individuals of the current investigation. Restricting the analysis to a cohort of obese individuals, as in the present study, age remains a significant deter- 

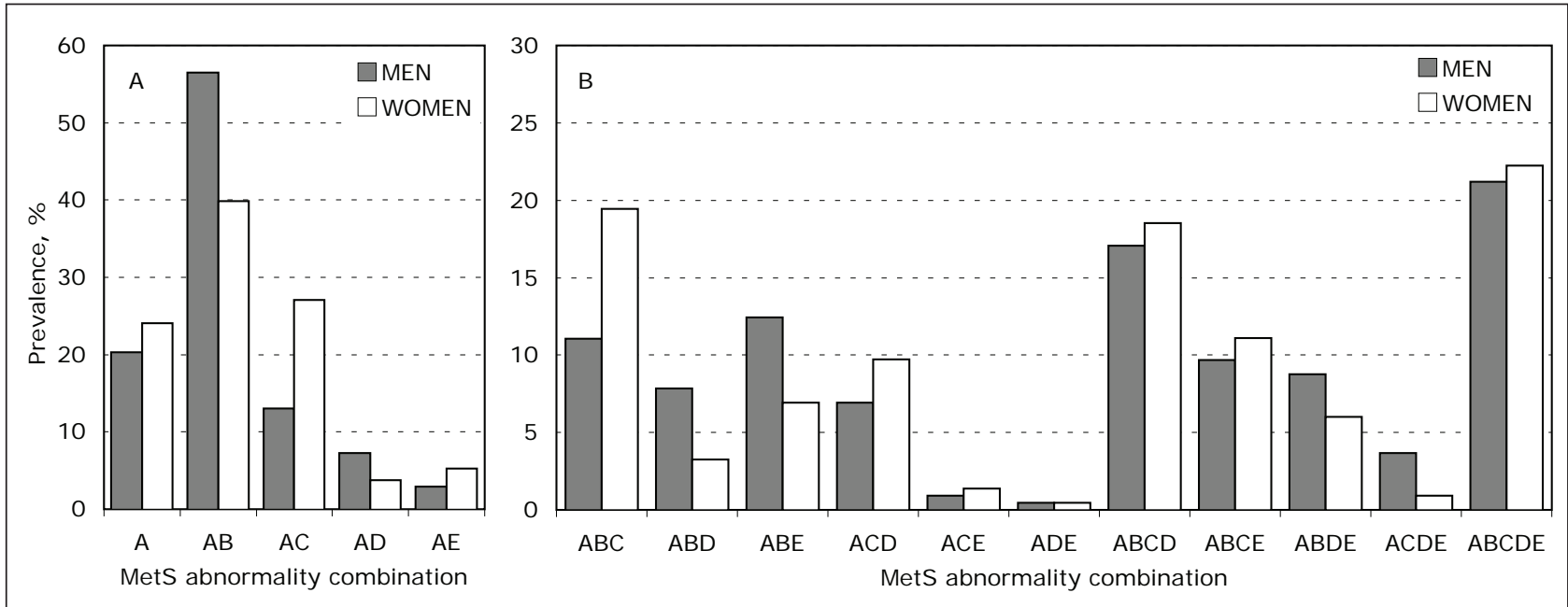

Fig. 2. The prevalence of the different combinations of abnormalities of the metabolic syndrome (MetS) in men and women among individuals $\mathbf{A}$ without and $\mathbf{B}$ with the MetS. A = central obesity; $\mathrm{B}=\operatorname{raised}$ blood pressure or treatment of previously diagnosed hypertension; $\mathrm{C}=$ reduced HDL cholesterol or specific treatment for this lipid abnormality; D = raised triglycerides level or specific treatment for this lipid abnormality; $\mathrm{E}$ = raised fasting plasma glucose concentration or previously diagnosed type 2 diabetes.

minant of MetS independent of the degree of obesity, as evidenced by the results of the multivariate binary logistic regression analysis (table 2). Although no clear age-dependent trend in the prevalence of low HDL-CH levels was detected, the other individual metabolic abnormalities (raised BP, TG and FPG) markedly increased with age (fig. 1A, B). Similar trends in the prevalence of individual components of the MetS with advancing age have also been reported in white individuals from a cross-sectional survey on the US national population [7]. Ageing per se has been found to be associated with a number of metabolic derangements resulting from reduced pancreatic cell function and sarcopenic processes, whose mechanisms are not completely clear but are ultimately responsible for age-related insulin resistance, type 2 diabetes mellitus, dyslipidaemia, and hypertension, with a possible contribution also of the decreased physical activity $[15,16]$. Thus, present data confirm that, also among the obese population, the contribution of age to the development of the MetS is partially based on aspects that could be considered independent of obesity-related factors.

Compared with studies conducted on large samples of the general population from different Italian regions, which document an overall prevalence around $17-18 \%[3,17]$, the figures reported here illustrate the dramatic effect of obesity on the metabolic functions and closely agree with previous estimates obtained with the same IDF criteria among obese females in Italy [10] and Portugal [18]. Nonetheless, it should be recognised that our investigation might possibly have overestimated the actual prevalence of the MetS in obesity since the obese individuals of the study were seeking treatment for their condition and some metabolic abnormalities may have selectively stimulated them to ask medical help.

Obesity is considered the central causative factor in the development of the MetS as adipose cells produced bioactive substances directly influencing insulin sensitivity and vascular injury $[8,9]$. In fact, the degree of obesity, as evaluated through BMI, has been found to contribute in a dramatic way to the development of the MetS in the US national population [7], with a prevalence of about $60-70 \%$ and $50-65 \%$ within the obese groups of men and women with BMI $\geq 35 \mathrm{~kg} / \mathrm{m}^{2}$, respectively. Nonetheless, to our knowledge, no study has 


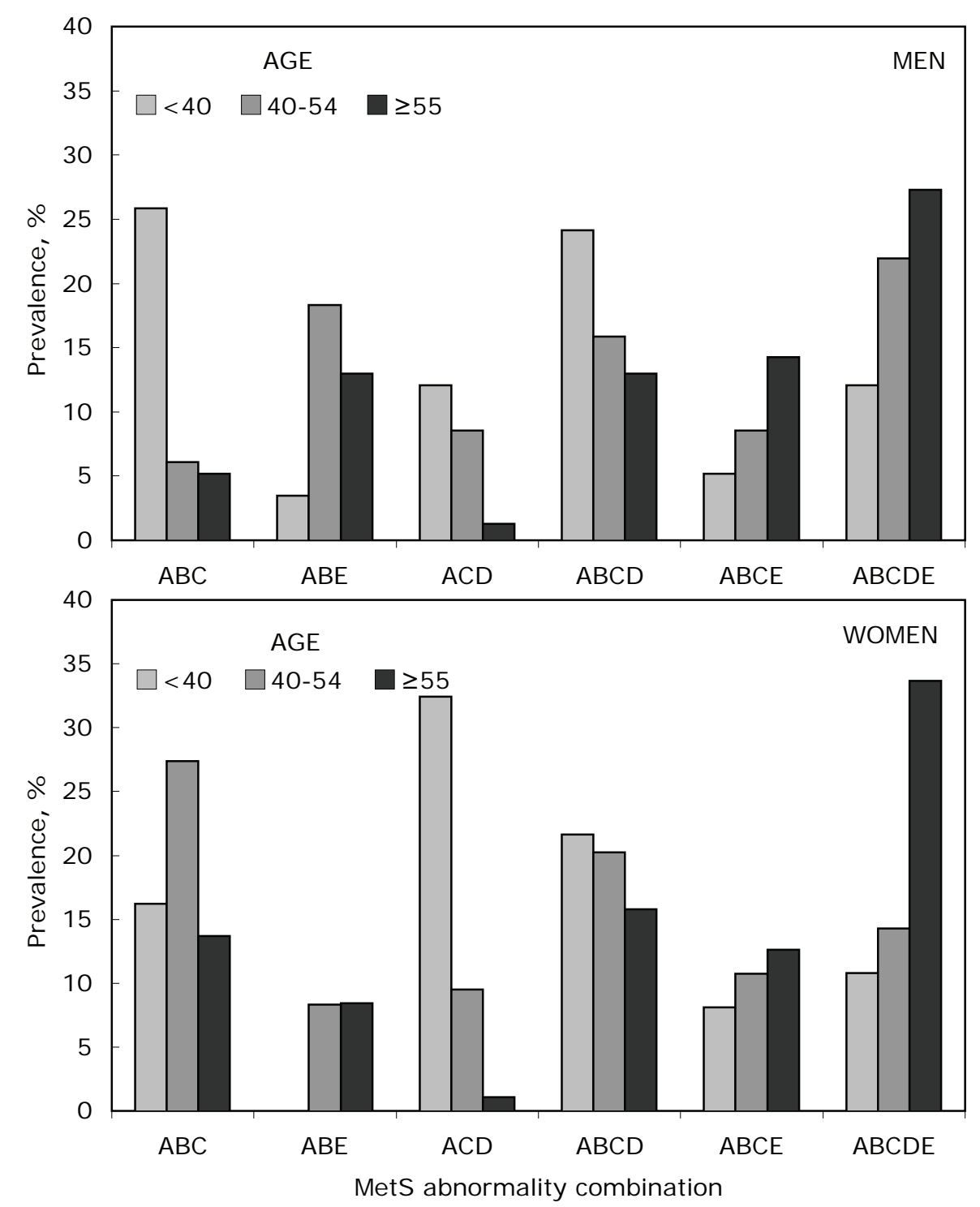

Fig. 3. The prevalence of the principal combinations of abnormalities of the metabolic syndrome within the different strata of age for affected $\mathbf{A}$ men and $\mathbf{B}$ women. $\mathrm{A}=$ central obesity; $\mathrm{B}=$ raised blood pressure or treatment of previously diagnosed hypertension; $\mathrm{C}$ = reduced HDL cholesterol or specific treatment for this lipid abnormality; D = raised triglycerides level or specific treatment for this lipid abnormality; $\mathrm{E}=$ raised fasting plasma glucose concentration or previously diagnosed type 2 diabetes.

been previously performed on restricted samples of obese individuals of both genders. Interestingly, when the analysis is restricted to obese persons only, as in the present investigation, no effect of BMI is detectable on the MetS prevalence, indicating that the degree of obesity per se scarcely plays a role in the development of the MetS among obese people. In contrast, the multivariate analysis revealed that both high WC and high WHR appear to be important independent determinants of the MetS in obese women, but not in men.

Thus, MetS seems to have a different profile in obese men and women, possibly deriving from different sex hormone levels influencing regulatory mechanisms of metabolism. 
Indeed, there is abundant epidemiological evidence that central fat distribution is a clear risk factor for development of diabetes and cardiovascular disease in obese as well as in normal-weight individuals [19]. Therefore, since adipose tissue accumulates with a genderspecific, hormonally regulated pattern, men are more prone to central obesity and the related effects of adipokines on lipid metabolism, glucose homeostasis and vascular injury $[8,9]$; lower deposition of visceral fat may be an important determinant of the reduced tendency to develop MetS that we observed in women. Nonetheless, the molecular basis for the different effect of WC and WHR on the MetS prevalence among men and women is unclear.

Beside the difference in absolute MetS prevalence, also in its components a genderrelated distribution can be detected. Hypertension was the most common abnormality in both genders, although significantly less prevalent in women (table 2), and is possibly related to differences in central fat distribution, renin-angiotensin system activation and salt sensitivity in BP regulation [12]. However, with advancing age, high BP prevalence in these women sharply increased (from $33.3 \%$ in the youngest group to $92.5 \%$ in the age group $>55$ years), attaining thus values comparable to those of age-matched men $(91.3 \%$, fig. 1A, B). Indeed, the increased prevalence of hypertension post menopause in population surveys is well known [20]. Moreover, high FPG and high TG were detected to have greater prevalence in obese males than females of the present cohort, which is in line with general population trends [21]. Similarly, lower TG and higher HDL-CH levels observed in premenopausal women have been interpreted to be due, at least in part, to the effect of oestrogens [22], although we failed to detect any significant difference in prevalence of low HDL-CH between men and women.

The present investigation also revealed wide variations in the prevalence of the combinations of individual metabolic abnormalities which determine the MetS (fig. 2,3). Although the full-blown picture of the MetS with all five abnormalities had similar prevalence in men and women, among individuals with a minimal profile of the condition with three abnormalities, the leading combination for men was central obesity-hypertension-hyperglycaemia, while for women it was central obesity-hypertension-low HDL-CH. A similar trend was observed also among those with two abnormalities (without MetS), the prevailing combination being central obesity-hypertension for men and central obesity-low HDL-CH for women (fig. 2). This pattern is consistent with the hierarchical classification model of Dallongeville et al. [23] who identified low HDL-CH among the factors with a significantly higher effect in women than in men, while BP was among the more important in men.

It is interesting to notice that those combinations including raised FPG occurred in significantly older patients and their prevalence increased progressively in subsequent strata of age (fig. 3). It could be hypothesised that these combinations may represent different stages in the evolution of the syndrome, with FPG concentration in the normal range and raised FSI at an earlier stage and in younger patients and increasing levels of hyperglycaemia at a later stage and in older patients [24].

All principal combinations of abnormalities we have detected in this report also included high BP, apparently due to the high prevalence of hypertension in obesity. Nonetheless, in a recent study on a cohort of obese women, we revealed by means of a factor analysis that systolic and diastolic BP are segregated as a separate factor, without association with insulin resistance, hyperglycaemia and dyslipidaemia [11]. This should indicate that, at least in females, BP regulation is chiefly mediated by other separate physiological processes [25], despite the highly significant contribution of hypertension to the MetS. Thus, the mechanisms leading to the combination of the different abnormalities still appear unclear and need to be elucidated.

Although sex differences in cardiovascular risk associated with the MetS are being recognised [12], it is not known if, among affected men and women, different combinations 
of abnormalities are necessarily associated also with different risks, and further investigation seems to merit consideration. In fact, based on present data, it is not possible to estimate the expected progression with time and the inherent relative risk or, conversely, the potential reversibility on treatment.

These issues have considerable importance within the strategies of prevention and treatment of the MetS in obese individuals. In spite of the alarmingly high prevalence of the MetS among obese individuals, rapid improvements in single abnormalities are observed after even small weight loss [26, 27]; Sartorio et al. [28] reported a 16\% short-term decrease in cardiovascular risk score after a weight loss of about $5 \%$ in severely obese subjects.

Thus, a better understanding of the mechanisms responsible for sex differences in MetS manifestations could be relevant in practical approaches aiming at prevention and treatment of the syndrome.

\section{Disclosure Statement}

The authors declare that there is no conflict of interest that could be perceived as prejudicing the impartiality of the research reported.

\section{References}

1 Reynolds K, He J. Epidemiology of the metabolic syndrome. Am J Med Sci 2005;330:273-279.

2 Cameron AJ, Shaw JE, Zimmet PZ: The metabolic syndrome: prevalence in worldwide populations. Endocrinol Metab Clin North Am 2004;33:351-375.

3 Miccoli R, Bianchi C, Odoguardi L, Penno G, Caricato F, Giovannitti MG, Pucci L, Del Prato S: Prevalence of the metabolic syndrome among Italian adults according to ATP III definition. Nutr Metab Cardiovasc Dis 2005;15:250-254.

4 Mottillo S, Filion KB, Genest J, Joseph L, Pilote L, Poirier P, Rinfret S, Schiffrin EL, Eisenberg MJ: The metabolic syndrome and cardiovascular risk. A systematic review and meta-analysis. J Am Coll Cardiol 2010; 56:1113-1132.

5 Carr DB, Utzschneider KM, Hull RL, Kodama K, Retzlaff BM, Brunzell JD, Shofer JB, Fish BE, Knopp RH, Kahn SE: Intra-abdominal fat is a major determinant of the National Cholesterol Education Program Adult Treatment Panel III criteria for the metabolic syndrome. Diabetes 2004;53:2087-2094.

- 6 Alberti KG, Zimmet P, Shaw J: Metabolic syndrome - a new world-wide definition. A Consensus Statement from the International Diabetes Federation. Diabet Med 2006;23:469-480.

- 7 Park YW, Zhu S, Palaniappan L, Heshka S, Carnethon MR, Heymsfield SB: The metabolic syndrome: prevalence and associated risk factor findings in the US population from the Third National Health and Nutrition Examination Survey, 1988-1994. Arch Intern Med 2003;163:427-436.

- 8 Hutley L, Prins JB: Fat as an endocrine organ: relationship to the metabolic syndrome, Am J Med Sci 2005; 330:280-289.

- 9 Waki H, Tontonoz P: Endocrine functions of adipose tissue. Annu Rev Pathol 2007;2:31-56.

10 Sartorio A, Agosti F, Adorni F, Pera F, Lafortuna CL: Effect of age, degree and distribution of adiposity on the prevalence of the metabolic syndrome in a cohort of obese Italian women. Diabetes Res Clin Pract 2007; 78:225-233.

-11 Lafortuna CL, Adorni F, Agosti F, Sartorio A: Factor analysis of metabolic syndrome components in obese women. Nutr Metab Cardiovasc Dis 2008;18:233-241.

-12 Regitz-Zagrosek V, Lehmkuhl E, Weickert MO: Gender differences in the metabolic syndrome and their role for cardiovascular disease. Clin Res Cardiol 2006;95:136-147.

13 World Health Organization: Measuring obesity - classification and description of anthropometric data. Report on a WHO consultation on the epidemiology of obesity. EUR/ICP/NUT 125. Warsaw, WHO Regional Office for Europe. 1987.

14 Wallace TM, Levy JC, Matthews DR: Use and abuse of HOMA modeling. Diabetes Care 2004;27:1487-1495.

15 Chang AM, Halter JB: Aging and insulin secretion. Am J Physiol Endocrinol Metab 2003;284:E7-E12.

16 Karakelides H, Nair KS: Sarcopenia of aging and its metabolic impact. Curr Top Dev Biol 2005;68:123-148.

17 Bonora E, Kiechl S, Willeit J, Oberhollenzer F, Egger G, Bonadonna RC, Muggeo M: Metabolic syndrome: epidemiology and more extensive phenotypic description. Cross-sectional data from the Bruneck Study. Int J Obes Relat Metab Disord 2003;27:1283-1289. 
18 Correia F, Poínhos R, Freitas P, Pinhão S, Maia A, Carvalho D, Medina JL: Prevalence of the metabolic syndrome: comparison between ATPIII and IDF criteria in a feminine population with severe obesity (in Portugese). Acta Med Port 2006;19:289-293.

19 Pi-Sunyer FX: The epidemiology of central fat distribution in relation to disease. Nutr Rev 2004;62:S120 S126.

20 Joffres MR, Hamet P, MacLean DR, L'italien GJ, Fodor G: Distribution of blood pressure and hypertension in Canada and the United States. Am J Hypertens 2001;14:1099-1105.

21 Geer EB, Shen W: Gender differences in insulin resistance, body composition, and energy balance. Gend Med 2009;6:60-75.

22 Williams CM: Lipid metabolism in women. Proc Nutr Soc 2004;63:153-160.

-23 Dallongeville J, Cottel D, Arveiler D, Tauber JP, Bingham A, Wagner A, Fauvel J, Ferrières J, Ducimetière $\mathrm{P}$, Amouyel P: The association of metabolic disorders with the metabolic syndrome is different in men and women. Ann Nutr Metab 2004;48:43-50.

24 Accili D: Lilly lecture 2003:the struggle for mastery in insulin action: from triumvirate to republic. Diabetes 2004;53:1633-1642.

25 Reaven GM, Lithell H, Landsberg L: Hypertension and associated metabolic abnormalities: the role of insulin resistance and the sympathoadrenal system. N Engl J Med 1996;334:374-381.

-26 Diehl HA: Coronary risk reduction through intensive community-based lifestyle intervention: the Coronary Health Improvement Project (CHIP) experience. Am J Cardiol 1998;82:83T-87T.

27 Sjöström M, Karlsson AB, Kaati G, Yngve A, Green LW, Bygren LO: A four-week residential program for primary health care patients to control obesity and related heart risk factors: effective application of principles of learning and lifestyle change, Eur J Clin Nutr 1999;53:S72-S77.

28 Sartorio A, Lafortuna CL, Vangeli V, Tavani A, Bosetti C, La Vecchia C: Short-term changes of cardiovascular risk factors after a non-pharmacological body weight reduction program. Eur J Clin Nutr 2001;55:865-869. 